\title{
Estimating Intrinsic Parameter of Camera from a Single Image
}

[Geetha Kiran A. and Murali S.]

\begin{abstract}
In this paper, an intrinsic parameter of camera from a single image is formulated. Compared to other techniques which use two or three orthogonal planes, parallelograms, rectangles, conic circles and balls, the proposed technique is easy to use and flexible. Calculation of the focal length using a single image is the main objective of this research work. A stick is employed as the calibration object to formulate the equations in order to compute the focal length. The arrangement of the calibration object to acquire the images adds more accuracy in computing the focal length of the camera. The length of the calibration object is the major cue in computing the focal length. A novel approach using polynomial regression is attempted in formulating the cubic polynomials based on the length of the calibration object. The results are tabulated and further used in formulating equation to compute the focal length of the camera. Experimental results are tabulated to show the accuracy of the proposed approach.
\end{abstract}

Keywords-Polynomial Regression, Focal length, Calibration Object, Cubic polynomials

\section{Introduction}

Images are two dimensional representation of the three dimensional world. The drop from three dimensional world to a two dimensional image is a projection process in which there is a loss of one dimension as shown in Fig.1. The purpose of camera calibration is to establish the transformation between the object and the image space. With the aid of the transformation established, the object space information along with the other parameters of the camera can be inferred from image space. The camera calibration is identified to compute the intrinsic and extrinsic camera parameters. The extrinsic parameters of a camera indicate the position and the orientation of the camera with respect to a world-coordinate system. The intrinsic parameters characterize the inherent properties of the camera optics which includes the focal length, image center, the image scaling factor and the lens distortion coefficient.

\section{Geetha Kiran A}

Malnad College of Engineering

India

Murali S

Maharaja Institute of Technology India

A brief description of the imaging process is given: The perspective projection from a $3 \mathrm{D}$ point onto the image plane, and the back projection from an image pixel to 3D world knowing the depth of the point are related by the following formula:

$$
\left[\begin{array}{l}
u \\
v \\
1
\end{array}\right]=A\left[\begin{array}{ll}
R & T
\end{array}\right]\left[\begin{array}{l}
X \\
Y \\
Z \\
1
\end{array}\right]
$$

where $\left[\begin{array}{lll}u & v & 1\end{array}\right]^{\mathrm{T}}$ is the 2D point position in pixel coordinates, $\left[\begin{array}{llll}X & Y & Z & 1\end{array}\right]^{\mathrm{T}}$ represent a 3D point position in world coordinates, $A$ is the calibration matrix encoding the intrinsic camera parameters as given in equation (2) and $\mathrm{R}, \mathrm{T}$ are the extrinsic parameters which denote the coordinate system transformations from 3D world coordinates to 3D camera coordinates.

$$
\mathrm{A}=\left[\begin{array}{ccc}
\propto_{x} & \gamma & u_{0} \\
0 & \propto_{y} & v_{0} \\
0 & 0 & 1
\end{array}\right]
$$

where $\propto_{x}=\mathrm{f} . \mathrm{m}_{\mathrm{x}}$ and $\propto_{y}=\mathrm{f} . \mathrm{m}_{\mathrm{y}}$ are the focal lengths of the camera in pixels, $\mathrm{m}_{\mathrm{x}}$ and $\mathrm{m}_{\mathrm{y}}$ are the scale factors relating pixels to the distance and $\mathrm{f}$ is the focal length in terms of distance, $\left(u_{0}, v_{0}\right)$ is the principal point of the image plane and $\gamma$ is the skew parameter of the camera.

A novel method of calibrating the intrinsic parameter i.e., the focal length and the extrinsic parameter i.e., the position of the camera using polynomial regression is attempted in the proposed work. This fits a polynomial regression model to powers of a single predictor by the method of linear least squares. The premise of polynomial regression is that a data set of $n$ paired $(x, y)$ members: $\left(x_{1}, y_{1}\right) \ldots \ldots \ldots . .\left(x_{n}, y_{n}\right)$ can be processed using a least squares method to create a predictive polynomial equation of degree $\mathrm{p}$ :

$$
\mathrm{Y}=\mathrm{a}_{0}+\mathrm{a}_{1} \mathrm{x}+\ldots \ldots \ldots \ldots \ldots \ldots+\mathrm{a}_{\mathrm{p}} \mathrm{x}^{\mathrm{p}}, \quad \mathrm{p}<\mathrm{n} .
$$

The essence of the method is to reduce the residual $\mathrm{R}$ at each data point:

$$
\mathrm{R}_{\mathrm{i}}=\mathrm{y}_{\mathrm{i}}-\mathrm{a}_{0}-\mathrm{a}_{1} \mathrm{x}_{\mathrm{i}}-\mathrm{a}_{2} \mathrm{x}_{\mathrm{i}}^{2} \ldots \ldots \ldots \ldots \ldots \ldots-\mathrm{a}_{\mathrm{p}} \mathrm{x}_{\mathrm{i}}^{\mathrm{p}} .
$$

The computed $\mathrm{R}$ is used in predicting the better choice of the model.An attempt to achieve the accuracy of the adopted method is presented with detailed result analysis.

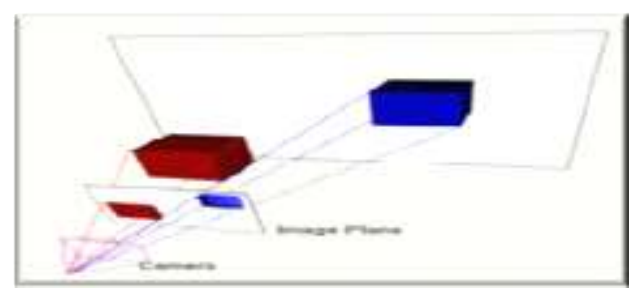

Figure. 1. 3D object projected on to a 2D plane

Photogrammetry is the process of determining the geometric properties of objects from photographic image. As the 
profession evolves and the technology advances, the calibration task becomes more complex. The quality of camera systems has improved significantly over the years and one might argue that camera calibration may appear to be less important. However, the development of new sensors and integration of these sensors with other systems make the need for calibration as important as before and probably more difficult to perform. This motivated to estimate the camera parameters with a novel approach using polynomial regression which lessens the task compared to the existing methods. Polynomial Regression fits a polynomial model with one predictor variable which facilitates the need for the proposed work.

The organization of the paper is as follows: Section II presents some of the related works. In section III, the steps taken in creating the dataset is endeavored. Section IV briefly explains polynomial regression and its application in computing the equations for the focal length. Section $\mathrm{V}$ gives the algorithm followed by Results and Analysis and concluded in Section VI.

\section{Related Works}

Calibrating camera from a single image requires prior information of the scene. It is learnt from the literature that no single calibration technique suits best for all the applications, as the application depends on the situation a user needs to deal with. Few of the existing methods are discussed here. Classical camera calibration methods require more than one image, they partly depend on the relationship between images. Since camera calibration is essential for 3D reconstruction, classical methods lack the power to deal with single images from unknown sources such as the Internet or magazines. Another direction is camera calibration from a single image. Unlike camera calibration from multiple images, for a single image, prior geometrical information is required. The related works regarding the single camera calibration is discussed below.

The different approaches in attaining the camera calibration can be classified in the following categories:

- Using Vanishing points and Vanishing lines

- Using Circles

- Using Collaboration of Vanishing point/lines and circles

- Using Symmetry

- Using four coplanar control lines

- Using three known coplanar points

The method proposed by Caprile and Torre [1] is based on the view of a cube, whose orientation is set at $45^{\circ}$ in order to achieve optimized accuracy. Three vanishing points can be retrieved from the image of the cube and the intrinsic parameter can be calculated from the property of the vanishing points. Guillou et al. [2] extended the work without any calibration pattern using a single image containing two vanishing points, a known line segment, the principal point of which lies in the center of the image and the user-fixed aspect ratio. The work of Svedberg and carlsson [3] is also derived from Caprile and Torre using the seemingly orthogonal wedge. One more work by Wilczkowiak [6] using parallelepipeds rather than cubes is claimed to be an improvement since computing vanishing points is numerically unstable. The other works proposed by
Deutschu [4], Kushaln et al. [5] and Wilczkowiak et al. [6] is an improvement by adding the ideas of normalization and factorization, the intrinsic parameters can be computed and the error can be suppressed. Wu et al. [7] adopted RANSAC algorithm along with vanishing points and lines. The latest work on this approach is by Avinash and Murali [8] who propose a method of containing a rectangular prism. The rectangular prism is used to generate two vanishing points. Then fixing up the picture plane and fixing up the station point are carried out. Thus the intrinsic parameters can be calculated from the formed equation. Frenment and Chellali [9] uses one image of two concentric circles of known radii. The perspective projections of concentric circles i.e., ellipses are estimated to find the intrinsic and extrinsic parameters. Teramoto and $\mathrm{Xu}$ [10] have proposed a method which contains 3 balls. Chen et al [11] calibrate camera with two coplanar circles. Jiang and Quan[12] propose a way of detecting concentric circles and form a correspondent calibration method. Colombo et al. calibrate the camera with two coaxial circles. Wang et al. [13] contributed camera calibration from two line segments with equal length or known length ratio, circle and a vanishing point. Chen et al. [14] calculates all intrinsic parameters from two coplanar circles and vanishing points/lines. The use of symmetry was proposed by Hong et al. [15] using translational, reflective and rotational symmetry. Shang et al adopt 4 coplanar lines in the image. The authors claim that using control lines also brings the benefit of suppressing the image noise.

In spite of the existing ample number of techniques found in the literature for camera calibration, still there is a scope for a stable self-calibration method in real time conditions. The linear calibration techniques use reference points of the reference object in the world coordinate system [16]. In case of mobile robots and autonomous vehicles, as the camera will be in motion, mapping of the world coordinate system and the camera coordinate system is a challenging task. The existing works use multi-point correspondence. Motivated by these factors, the current work attempts in designing the technique in finding the camera parameters based on the length of the calibration object. An additional strength of the proposed work is to achieve the calibration with the two-point correspondence. A basic foundation for camera calibration with low computational complexity is achieved. In the current work, the focus is on finding the intrinsic parameter (Focal Length). A novel approach is designed using polynomial equations of order 3 and a stick as the calibration object in the current work.

\section{Generating Dataset}

Images of the chosen calibration object (i.e., stick) are captured using Nikon camera of resolution 3872 × 2592. The actual length of the calibration object is $25 \mathrm{~cm}$. The image of the calibration object is captured by aligning it, in line with the lens of the camera at six different focal lengths $(18 \mathrm{~mm}, 24 \mathrm{~mm}$, $35 \mathrm{~mm}, 50 \mathrm{~mm}, 70 \mathrm{~mm}$ and $105 \mathrm{~mm}$ ). At each focal length, the distance of the calibration object is varied from $50 \mathrm{~cm}$ to $160 \mathrm{~cm}$ in steps of $10 \mathrm{~cm}$. For each distance, the calibration object is varied at the angular orientation of $10^{\circ}$ each starting from $0^{\circ}$ to $90^{\circ}$. The process is repeated at all levels of distances and focal lengths. The length of the calibration object is measured in 
pixels from each captured image and thus a data set is created as shown in TABLE III.

The length of the stick measured at varied distances facilitating the prerequisites to compute the focal length of the camera using polynomial regression is explained in the section to follow.

To compute the focal length of the camera, the steps taken are:

1. Constructing Polynomial equations for distances and angles.

2. Building the mathematical model based on the coefficient of the polynomial equations of step 1 .

3. Determining the intrinsic camera parameter- focal length.

These steps ultimately compute the focal length of the camera. The focal length of the lens is the distance between the lens and the image sensor when the subject is in focus, usually stated in millimeters (ex. $18 \mathrm{~mm}, 24 \mathrm{~mm}, 35 \mathrm{~mm}$ etc.). The angle of view is the visible extent of the scene captured by the image sensor. Wide angle of view capture greater areas, small angles capture smaller areas. Changing the focal length changes the angle of view. If the focal length $(18 \mathrm{~mm})$ is shorter, the angle of view will be wider and greater area will be captured. The longer the focal length $(105 \mathrm{~mm})$, the smaller the angle and the subject appears to be large. Fig. 2 illustrates the above discussed concepts.

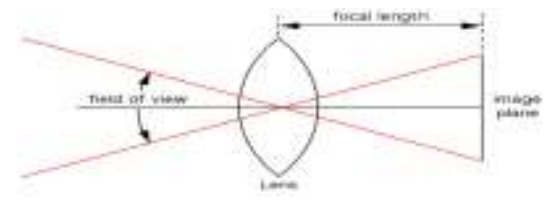

Figure 2. Illustrating relationship between focal length and the field of view

\section{Iv. Polynomial Regression Analysis}

The previous section gives the necessary setup of the calibration object required for the image acquisition and the dataset of the length of the stick facilitating the need in computing the focal length of the camera. The dataset is analyzed with the polynomial regression as explained below to find the camera parameter. A statistical method called "regression" is used to estimate the unknown values of one variable from the known values of other variable. Polynomial regression is a form of linear regression in which the relationship between the independent variable $\mathbf{x}$ and the dependent variable $\mathbf{y}$ is modeled as an $\mathrm{n}^{\text {th }}$ order polynomial. Polynomial regression fits a nonlinear relationship between the value of $\mathbf{x}$ and the corresponding conditional mean of $y$, denoted $\mathrm{E}(\mathrm{y} / \mathrm{x})$. The goal of regression analysis is to model the expected value of a dependent variable $y$ in terms of the value of an independent variable $\mathrm{x}$.

In simple linear regression, the model

$$
\mathrm{Y}=\mathrm{a}_{0}+\mathrm{a}_{1} \mathrm{x}+€
$$

where $€$ is an unobserved random error. In this, for each unit increase in $\mathrm{x}$, the conditional expectation of $\mathrm{y}$ increases by $\mathrm{a}_{1}$ units.

In case of quadratic model, it takes the form:

$$
Y=a_{0}+a_{1} x+a_{2} x^{2}+a_{3} x^{3}+€
$$

In this, for each unit increase in $\mathrm{x}$, the conditional expectation of $y$ increases by $a_{1}+2 a_{2} x$ units.

In general, the expected value of $y$ has an $n^{\text {th }}$ order polynomial,

$$
\mathrm{Y}=\mathrm{a}_{0}+\mathrm{a}_{1} \mathrm{x}+\mathrm{a}_{2} \mathrm{x}^{2}+\mathrm{a}_{3} \mathrm{x}^{3+} \ldots+\mathrm{a}_{\mathrm{n}} \mathrm{x}^{\mathrm{n}}+€
$$

In statistics, the coefficient of determination $R^{2}$ is the proportion of variability in a dataset that is accounted by a statistical model. In this definition, the term 'variability' is defined as the sum squares. Coefficient of determination is used in trend analysis. It is computed as a value between $0(0$ percent) and 1 (100 percent). When the value is higher, it will fit better. Coefficient of determination is symbolized by $\mathrm{R}^{2}$ because it is square of the coefficient of correlation symbolized by $R$. The coefficient of determination is important in determining the degree of linear-correlation of variables ('goodness of fit') in regression analysis and is called R-square.. $\mathrm{R}$-square always increases when a new term is added to a model. The result of polynomial regression analysis at each distance varied at different angles with the R-Square is given in TABLE IV. In the experiment conducted, the $\mathrm{R}^{2}$ varies from $99.8 \%$ to $100 \%$.

The equations generated are of the form:

$$
\mathrm{Y}=\mathrm{a}+\mathrm{bx}+\mathrm{cx^{2 }}+\mathrm{dx} \mathrm{x}^{3}
$$

where $a$ is the initial intercept; b, c and $d$ are the partial regression coefficients for variables $x, x^{2}$ and $x^{3}$. The general principles of polynomial regression are the fitted model and is more reliable when it is built on large number of observations and do not extrapolate beyond the limits of observed values. Also choose values for the predictor (x) that is not too large as they will cause overflow with high degree polynomial.

Using the constants $\mathrm{a}, \mathrm{b}, \mathrm{c}$ and $\mathrm{d}$ from the regression equation (Refer TABLE IV -Distance $100 \mathrm{~cm}$ ) the mathematical equations (9,10 and 11) are formed. The mathematical equations for the other distances are also derived.

TABLE I. CONSTANTS USED IN DERIVING EQUATIONS AT Distance 100CM

\section{Distance $=100 \mathrm{~cm}$}

\begin{tabular}{|c|c|c|c|c|}
\hline & $\mathrm{a}$ & $\mathrm{b}$ & $\mathrm{c}$ & $\mathrm{d}$ \\
\hline 18 & 730.5 & -3.024 & -0.094 & 0 \\
\hline 24 & 975.1 & -3.435 & -0.145 & 0 \\
\hline 35 & 1358 & -4.364 & -0.217 & 0.001 \\
\hline 50 & 1914 & -7.392 & -0.273 & 0.001 \\
\hline 70 & 1338 & 48.3 & -1.275 & 0.006 \\
\hline
\end{tabular}

Polynomial regression equation is generated for each of the constant as below:

For constant $b$,

$\mathrm{FL}=5.289-4.823 * \mathrm{x}+0.183 * \mathrm{x}^{2}-0.001 * \mathrm{x}^{3}$

For constant c,

$\mathrm{FL}=-5.489-246.6 * \mathrm{x}-216.4 * \mathrm{x}^{2}-54.46 * \mathrm{x}^{3}$ 
For constant d,

$\mathrm{FL}=21+25515 * \mathrm{x}-4 * 10^{6} * \mathrm{x}^{2}+2 * 10^{8} * \mathrm{x}^{3}$

These equations $(9,10$ and 11$)$ are used to compute the intrinsic camera parameter (Focal length).

\section{v. Algorithm/ Results and Analysis}

The steps taken to find the focal length of the camera are:

1. An image of the calibration object at any orientation, distance and focal length is given as an input.

2. The length and angle of the calibration object is computed.

3. Computed length in step 2 is compared with the dataset and the corresponding polynomial regression equation coefficients (b, c, d) are retrieved.

4. The partial regression coefficients (b, c, d from TABLE I) are applied to the computed mathematical equations ((9), (10)and (11)).

5. The focal length is computed using all the three equations (((9), (10) and (11)).

6. The average of the focal length obtained in step 5 gives the focal length of the camera.

The proposed method has been tested on the real images.

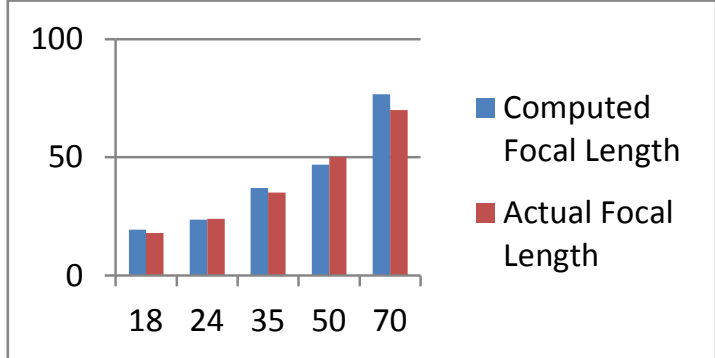

Figure3. Graph of AFL v/s CFL

The error obtained (TABLE II) and the graph (Fig. 3) shows the accuracy in the results. The error rate is analyzed by comparing the actual focal length (AFL) with the computed focal length (CFL) and found to be satisfactory with an average error rate of 1.340496 .

TABLE II. COMPARISON OF CFL WITH AFL

\begin{tabular}{|c|c|c|}
\hline CFL & AFL & Error \\
\hline 19.46646194 & 18 & 1.466462 \\
\hline 23.64667054 & 24 & -0.35333 \\
\hline 37.00315156 & 35 & 2.003152 \\
\hline 46.95724732 & 50 & -3.04275 \\
\hline 76.62894777 & 70 & 6.628948 \\
\hline
\end{tabular}

\section{Conclusion}

The paper presents a method to compute the intrinsic camera parameter based on the cubic polynomials using an easily available stick as a calibration object. The total number of images used for the experimentation is 720 images. The results obtained in the proposed work are accurate with an average error rate of 1.340496. The method has the advantage of being analytical and straight forward, comparing the results obtained with the zhang's method. It is non-iterative and consumes less time. One more advantage of the proposed work is that it requires only one image and the manual selection of only two points; in contrast, zhang's method requires several images, selecting seal the corner points of the checker board for each image which are used in the iterative solution. The major drawback of the proposed method is, it requires a specific setup to work accurately.

\section{References}

[1] Caprile, Bruno, and Vincent Torre. "Using vanishing points for camera calibration." International journal of computer vision 4.2 (1990): 127-139.

[2] Guillou, Erwan, et al. "Using vanishing points for camera calibration and coarse 3D reconstruction from a single image." The Visual Computer 16.7 (2000): 396-410.

[3] Svedberg, Daniel, and Stefan Carlsson. "Calibration, pose and novel views from single images of constrained scenes." Pattern Recognition Letters 21.13 (2000): 1125-1133.

[4] Deutscher, Jonathan, Michael Isard, and John MacCormick. "Automatic camera calibration from a single manhattan image." Computer Vision-ECCV 2002. Springer Berlin Heidelberg, 2002. $175-188$

[5] Kushal, Akash, Vikas Bansal, and Subhashis Banerjee. "A Simple Method for Interactive 3D Reconstruction, Camera Calibration from a Single View." ICVGIP. 2002.

[6] Wilczkowiak, Marta, Peter Sturm, and Edmond Boyer. "Using geometric constraints through parallelepipeds for calibration and 3D modeling." Pattern Analysis and Machine Intelligence, IEEE Transactions on 27.2 (2005): 194-207.

[7] Wu, Qi, Te-Chin Shao, and Tsuhan Chen. "Robust self-calibration from single image using RANSAC." Advances in Visual Computing. Springer Berlin Heidelberg, 2007. 230-237.

[8] Avinash, N., and S. Murali. "Perspective geometry based single image camera calibration." Journal of Mathematical Imaging and Vision 30.3 (2008): 221-230.

[9] Frémont, Vincent, and Ryad Chellali. "Direct camera calibration using two concentric circles from a single view." International Conference on Artificial Reality and Telexistence. 2002.

[10] Teramoto, Hirohisa, and Gang Xu. "Camera calibration by a single image of balls: From conics to the absolute conic." Proc. of 5th Asian Conference on Computer Vision. 2002.

[11] Chen, Qian, Haiyuan Wu, and Toshikazu Wada. "Camera calibration with two arbitrary coplanar circles." Computer VisionECCV 2004. Springer Berlin Heidelberg, 2004. 521-532.

[12] Jiang, Guang, and Long Quan. "Detection of concentric circles for camera calibration." Computer Vision, 2005. ICCV 2005. Tenth IEEE International Conference on. Vol. 1. IEEE, 2005.

[13] Wang, Guanghui, et al. "Camera calibration and 3D reconstruction from a single view based on scene constraints." Image and Vision Computing 23.3 (2005): 311-323.

[14] Chen, Yisong, et al. "Full camera calibration from a single view of planar scene." Advances in Visual Computing. Springer Berlin Heidelberg, 2008. 815-824.

[15] Hong, Wei, et al. "On symmetry and multiple-view geometry: Structure, pose, and calibration from a single image." International Journal of Computer Vision60.3 (2004): 241-265.

[16] Zhang Z, "A flexible new technique for camera calibration", Transactions on Pattern Analysis and Machine Intelligence", 22(11) IEEE,pp.1330-1334, 2000. 
Proc. of The Second Intl. Conf. On Advances In Computing, Control And Networking - ACCN 2015 Copyright $(\odot$ Institute of Research Engineers and Doctors, USA .All rights reserved. ISBN: 978-1-63248-073-6 doi: 10.15224/ 978-1-63248-073-6-76

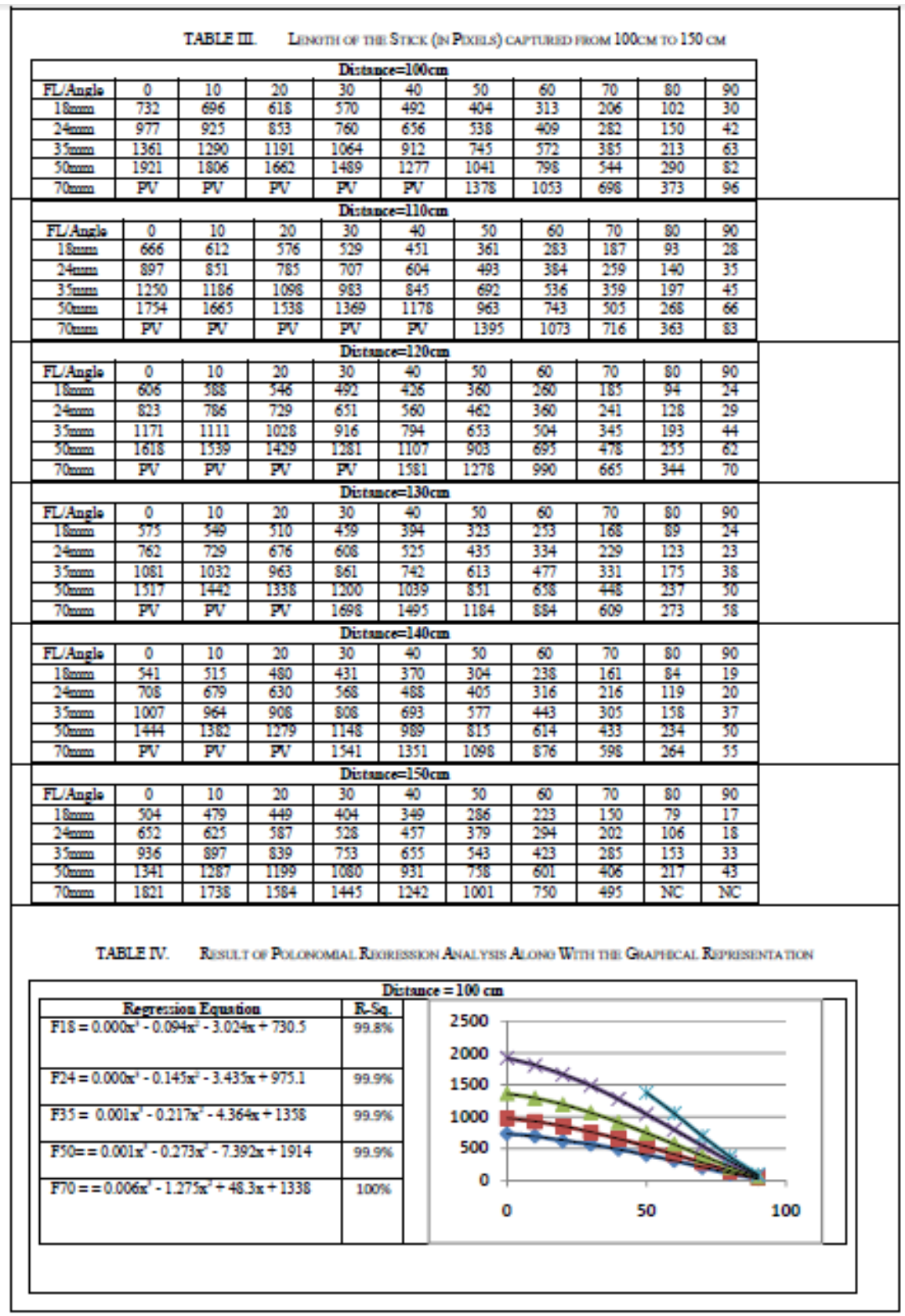

